\title{
Apport de Mycobacterium vaccae dans la Prévention et le Traitement de Certaines Maladies : Revue Bibliographique
}

\begin{abstract}
Ilham Zahir, Mohamed Karym, Fatima Ezzahra Ouatat, Rachid Essalhi, Laboratoire Polyvalent en Recherche et Développement, Université Sultan Moulay Sliman, Faculté Polydisciplinaire de Béni Mellal, Mghila, Béni Mellal, Maroc

Doi: 10.19044/esj.2019.v15n12p197 URL:http://dx.doi.org/10.19044/esj.2019.v15n12p197

\section{Résumé}

Ces dernières décennies, le rôle de Mycobacterium vaccae, une bactérie non pathogène et vivant naturellement dans le sol, comme modulatrice de l'immunité a été évoqué par plusieurs investigations. Ainsi, son apport dans la prévention et le traitement de nombreuses maladies telles que la tuberculose, la lèpre, l'allergie et l'asthme dans lesquelles l'immunité occupe une grande place a été mis en évidence. De telles pathologies présentent un fardeau pour la santé humaine et exigent pour leur traitement et surveillance l'administration des médicaments possédant une pléthore d'effets secondaires. Dans ce sens, l'effet des préparations à la base de Mycobacterium vaccae administrées sous différentes formes a été évalué sur des modèles murins ou dans des essaies cliniques. Il semble, dans le cas des maladies inflammatoires par exemple, que cette mycobactérie renforce l'immunité par l'augmentation de la réponse immunitaire de type $\mathrm{T}$ auxiliaires 1 (Th1), l'induction des lymphocytes T régulateurs, menant par la suite à la suppression de cellules de type TH2 et leurs médiateurs impliqués dans l'apparition de l'inflammation ce qui entraine, par conséquent, l'atténuation des symptômes pathologiques. Subséquemment, la présente synthèse bibliographique a pour objectif d'exposer l'impact de la bactérie en question pour lutter contre certaines maladies.
\end{abstract}

Mots-clés : Agent immuno-thérapeutique, lutte contre les maladies, modulation de l'immnité, Mycobacterium vaccae, réponse immunitaire de type $\mathrm{T}$ auxiliaires 1 


\title{
Contribution of Mycobacterium Vaccae in the Prevention and Treatment of Certain Diseases
}

\author{
Ilham Zahir, \\ Mohamed Karym, \\ Fatima Ezzahra Ouatat, \\ Rachid Essalhi, \\ Laboratoire Polyvalent en Recherche et Développement, \\ Université Sultan Moulay Sliman, Faculté Polydisciplinaire de Béni Mellal, \\ Mghila, Béni Mellal, Maroc
}

\begin{abstract}
In recent decades, the role of Mycobacterium vaccae, a nonpathogenic bacterium living naturally in the soil, as a modulator of immunity has been evoked by several investigations. Thus, its contribution in the prevention and treatment of many diseases such as tuberculosis, leprosy, allergy and asthma in which immunity occupies a large place has been highlighted. Such diseases present a burden on human health and require for their treatment and surveillance the administration of drugs with a plethora of side effects. In this sense, the effect of Mycobacterium vaccae preparations administered in different forms has been evaluated in murine models or in clinical trials. It seems, in the case of inflammatory diseases for example, that this mycobacterium enhances immunity by increasing the T helper 1 (Th1) immune response, inducing regulatory $\mathrm{T}$ cells, eventually leading to suppression of TH2-like cells and their mediators involved in the appearance of the inflammation which leads, therefore, to the attenuation of the pathological symptoms. Subsequently, this review aims to expose the impact of the bacterium in question to fight against certain diseases.
\end{abstract}

Keywords: Immunotherapeutic agent, disease control, modulation of immunity, Mycobacterium vaccae, T helper type 1 immune response

\section{Introduction}

Le dysfonctionnement du système immunitaire favorise le développement et la progression de plusieurs maladies plus ou moins graves à savoir l'asthme, le cancer, le psoriasis, la dermatite atopique, l'allergie, la lèpre et la tuberculose. D'autres maladies peuvent être davantage développées suite de cette anomalie. Incontestablement, une inflammation exagérée, à titre 
d'exemple, a été suggérée chez l'animal comme un facteur de risque de vulnérabilité au stress et de développement de comportements anxieux et de type dépressif, ainsi qu'une altération de l'apprentissage et de la mémoire (Fox et al., 2017). L'ensemble de ces maladies représentent un véritable problème pour la santé publique et un grand fardeau du point de vue souffrance humaine et coûts. Par ailleurs, les traitements actuellement disponibles peuvent atténuer les manifestations cliniques et les douleurs observées suite à ces pathologies mais au prix d'apparition des effets indésirables.

Dans ce sens, il faut chercher des adjuvants à des chimiothérapies existantes et/ou des alternatives non nuisibles à la santé humaine pour renforcer et aider le système immunitaire, dans la mesure du possible, afin de lutter contre les maladies précitées.

De là, il s'est émergé, pour gérer les maladies inflammatoires par exemple, l'idée de l'hypothèse hygiène qui stipule qu'une exposition environnementale précoce aux microorganismes non pathogènes et à leurs produits alimente les circuits immunorégulateurs en favorisant les réponses immunitaires innées et réduisant les maladies inflammatoires associées aux réponses de type $\mathrm{T}$ auxiliaires 2 (Th2) par l'augmentation des lymphocytes $\mathrm{T}$ régulateurs et la stimulations des cellules $\mathrm{T}$ auxiliaires 1 (Th1) (Racila et Kline, 2005 ; Le Bert et al., 2011 ; Akkoc et al., 2015 ; Fox et al., 2017). Subséquemment, un domaine actif et productif de la recherche actuelle étudie l'utilité des microbes et des produits microbiens pour la modulation de l'inflammation (Racila et Kline, 2005).

De la sorte, Mycobacterium vaccae, une mycobactérie atypique à croissance rapide non pathogène vivant naturellement dans sol, a été largement employé pour supporter cette hypothèse contemporaine (Boenickse et Juhasz, 1964 ; Le Bert et al., 2011) puisque il a été prouvé que ce microorganisme est un puissant inducteur de réponse immunitaire Th1 et des lymphocytes T régulateurs (Akkoc et al., 2015 ; Fox et al., 2017). En effet, l'immunothérapie à Mycobacterium vaccae s'est avérée bénéfique dans le traitement ou la prévention de nombreuses maladies à savoir l'asthme, l'allergie, le cancer, la lèpre, le psoriasis, la dermatite atopique, l'eczéma, la tuberculose, le stress et l'anxiété (Boenickse et Juhasz, 1964 ; (Stanford et al., 2009 ; Ho et al., 2012 ; Matthews et Jenks., 2013 ; Reber et al., 2016). En outre, il a été rapporté que des vaccins préparés à partir de $M$. vaccae modulent la réponse immunitaire durant les essaies cliniques. Plus intéressant encore, des études récentes ont montré que le contact avec $M$. vaccae est important pour le bon fonctionnement des circuits immuno-régulateurs affectant le comportement, l'émotivité et la santé (Matthews et Jenks., 2013). Ainsi, la présente revue bibliographique éclaircie l'impact de $M$. vaccae dans la prévention ou l'atténuation de la gravité de certaines maladies qui touchent la santé humaine. 


\section{Utilisation de $M$. vaccae dans la thérapie ou la prophylaxie de certaines maladies}

\section{1 - Asthme}

L'asthme est une maladie pulmonaire inflammatoire chronique complexe dans laquelle les éosinophiles, les lymphocytes $\mathrm{T}$, les macrophages, les mastocytes, les neutrophiles et les cellules épithéliales jouent un rôle clé dans la libération des médiateurs cellulaires, notamment des cytokines, et des facteurs de croissance, y compris, TGF- $\beta$, facteur de croissance endothélial vasculaire, et domaine 33 d'ADAM métallopeptidase, métalloprotéinase matricielle-9 et la famille de cytokines Th2 comprenant IL-5, 13 et 14. Ceuxci conduisent à une inflammation chronique prolongée des voies respiratoires qui déclenche une bronchoconstriction et un remodelage des voies respiratoires (Halwani et al., 2011; Al-Alawi et al., 2014). Les corticostéroïdes inhalés sont considérés comme le traitement antiinflammatoire de premier plan dans la gestion de l'asthme. Cependant, des effets indésirables à long terme de ces médicaments sont de plus en plus constatés (Bjermer et Diamant, 2004). En outre, ils sont inefficaces pour inverser le remodelage structurel des voies respiratoires (Halwani et al., 2011 ; Al-Alawi et al., 2014).

Cette réflexion a appelé à des alternatives de ce traitement, entre autres, figurent le traitement immunomodulateur avec $M$. vaccae guidant le système immunitaire vers une réponse immunitaire protectrice particulièrement forte de type T helper 1 (Th1) (Bjermer et Diamant, 2004 ; Yazi et al., 2007). En effet, maintes investigations ont suggéré un effet bénéfique du traitement et la prévention de l'asthme par M. vaccae (Zuany-Amorim et al., 2002; Camporota et al., 2003 ; Martinelli et al., 2004 ; Yazi et al., 2007). Prenant par exemple, celle menée par Yazi et ses collaborateurs en 2007 qui ont montré que l'administration intra-trachéale de $M$. vaccae a eu un effet d'amélioration durable sur les caractéristiques histo-pathologiques des voies respiratoires dans un modèle d'asthme murin. Ces observations ont motivé des chercheurs à effectuer une étude multicentrique de phase II, randomisée et contrôlée par placebo sur l'innocuité et l'efficacité de $M$. vaccae, tuée par la chaleur, dans le traitement de 178 patients adultes atteints d'asthme persistant modéré. Ainsi, une réduction des symptômes d'asthme a été observée chez les malades recevant deux doses élevées de $M$. vaccae administrées par voie intradermique. De plus, comparativement au placebo, $M$. vaccae, tuée par la chaleur, a significativement diminué les exacerbations de l'asthme (Rook et al., 2007). Afin de mettre en évidence le composant actif de la mycobactérie en question, une autre investigation utilisant une préparation de $M$. vaccae dépourvue des lipides n'a montré aucun effet du traitement (Shirtcliffe et al., 2003), montrant ainsi la nature lipidique éventuelles des molécules bio actives. 
Une étude plus remarquable dirigée par Li et ses collaborateurs (2016) a ciblé une autre méthode d'administration de $M$. vaccae afin de tester son effet sur la prévention de l'asthme chez des souris. Pour cette raison, la relation entre la nébulisation de $M$. vaccae et les cellules Th 9 , un type de lymphocytes $\mathrm{T} \mathrm{CD} 4^{+}$, chez des souris asthmatiques a été évaluée. Ainsi, dix-huit souris Balb / c ont été randomisées en 3 groupes de 6 souris chacun (groupe témoin normal, groupe témoin asthmatique et groupe asthmatique après nébulisation). Le groupe Neb a été nébulisé avec $M$. vaccae un mois avant l'établissement du modèle asthmatique avec sensibilisation à l'ovalbumine (OVA), et les groupes témoins normaux et asthmatiques ont été nébulisés avec une solution saline tamponnée au phosphate. Ainsi, l'inflammation des voies respiratoires a été atténuée, la réduction de la sécrétion de mucus et la diminution de l'hyperréactivité bronchique ont été également observées ainsi que les concentrations d'IL-9 et d'Ig E spécifiques de l'OVA ont été significativement réduits dans le groupe Neb par rapport au groupe témoin de l'asthme. Le même groupe Neb avait des pourcentages plus élevés de IL-10 par rapport au groupe contrôle asthmatique. Par conséquent, l'asthme bronchique de la souris pourrait être évité par la nébulisation de $M$. vaccae. Le mécanisme pourrait impliquer des effets médiés par $M$. vaccae sur l'induction de la sécrétion d'IL10 et la suppression de la sécrétion d'IL-9 par la régulation des cellules Th 9 (Li et al., 2016). Pour comprendre davantage ce mécanisme, il est crucial d'élucider certains points concernant la physiopathologie de l'asthme. En fait, il semble que certains lymphocytes $\mathrm{T}$ tels que les lymphocytes $\mathrm{T}$ auxiliaires (Th) et les lymphocytes $\mathrm{T}$ régulateurs ( $\mathrm{T}$ regs) affectent la durée de l'inflammation. Plus précisément, il y a une augmentation des cellules Th 2 et Th 17, et une diminution des cellules Th 1 et T reg dans l'asthme. En addition, les cellules Th 9, sécrètent principalement de l'interleukine IL -9 et de l'IL-10, qui sont étroitement liées à l'asthme. Indubitablement, l'IL-9 est une cytokine multi-effectrice agissant sur diverses cellules inflammatoires et tissulaires, produisant divers effets biologiques. Ainsi, il a été démontré que les cellules Th 9 sont directement liées à la sévérité de l'hyperréactivité bronchique et de l'inflammation des voies aériennes en favorisant les réactions allergiques, activant les mastocytes et agrégeant les manifestations pathologiques. D'un autre coté, l'IL-10, produite principalement par les cellules Th 9, Th 2 et T reg, est une cytokine suppressive importante, qui peut inhiber la prolifération et la différenciation des cellules T, ainsi que l'inflammation. En conséquence, la nébulisation de $M$. vaccae pourrait atténuer l'ensemble de réactions inflammatoires des voies respiratoires en agissant sur la sécrétion de l'IL -9 et de l'IL-10 (Li et al., 2016).

D’une manière similaire, à partir des modèles animales, il a été suggéré que la stimulation avec $M$. vaccae augmente l'expression des récepteurs appelés TLR2 (pour toll-like receptor), des protéines transmembranaires se 
trouvant à la surface des cellules présentatrices d'antigène (CPA) (Martinelli et al., 2004 ; Racila et Kline, 2005). En outre, l'engagement de TLR-2 a été également postulé comme un ligand important de lipoarabinomannane, une molécule glycolipidique de la paroi mycobactérienne (Racila et Kline, 2005 ; Zahir, 2018). Cette donnée rejoint ainsi les trouvailles de Shirtcliffe et al., 2003. Cette activation de TLR-2 contribue ainsi à la stimulation du système immunitaire inné de l'hôte et influence les réponses des cellules $\mathrm{T}$ par l'amélioration des réponses immunitaires à médiation cellulaire de type TH1 et l'induction des lymphocytes $\mathrm{T}$ régulateurs (Treg), conduisant par la suite à la suppression de cellules de genre TH2 et leurs médiateurs impliqués dans le schéma inflammatoire de l'asthme (Martinelli et al., 2004 ; Racila et Kline, 2005 ; Le Bert et al., 2011 ; Akkoc et al., 2015). Parmi ces médiateurs, on cite IL-5 qui active les éosinophiles et renforce leur adhésion aux cellules épithéliales vasculaires (Kikkawa et al., 2012). Par ailleurs, il a été supposé que les CPA CD11c+ pulmonaires induites par $M$. vaccae ont un rôle régulateur potentiel grâce à leur sécrétion de cytokines immuno-modulatrices dépendantes des TLR comme IL-10 et IFN- $\alpha$. (Martinelli et al., 2004 ). Il a été établi par Kikkawa et al., 2012 que IFN- $\alpha$. induit les cytokines TH1 et inhibe IL-5 menant ainsi à l'inhibition de recrutement des éosinophiles dans la paroi de la trachée et une amélioration de l'hypersensibilité des voies respiratoires chez les cobayes sensibilisés. L'IFN-alpha inhibe de manière significative l'adhésion des éosinophiles aux cellules endothéliales de la veine ombilicale humaine induites par l'IL-1 $\beta$.

\section{2 - Cancer}

En tenant en compte de l'utilisation de $M$. vaccae chez des patients présentant diverses pathologies, des effets remarquables ont été observés chez les patients atteints de cancers (Stanford et Stanford, 2012). Incontestablement, des études ont été réalisées dans lesquelles des malades présentant diverses tumeurs malignes ont reçu des injections intradermiques répétées. Ainsi, une amélioration significative de la qualité de vie (O'Brien et al., 2004), une meilleure tolérance aux effets secondaires de la chimiothérapie (Patel et al., 2008) et une augmentation de la survie ont été notées (Stanford et al., 2008). A titre d'exemple, une étude menée par Cananzi et al., (2013) a rapporté une survie à long terme meilleure chez 72 patients atteints de mélanome métastatique AJCC stade IV et qui ont reçu un vaccin à base de $M$. vaccae tuée par la chaleur. Un intérêt considérable dans la même préparation mycobactérienne a été également observé pour le traitement des cancers de la prostate, du poumon, du rein et de la peau. En particulier, cinq doses de $M$. vaccae ajoutées au cours de la chimiothérapie a augmenté la survie en moyenne de 4 mois et amélioré la qualité de vie dans les essais de phase III sur l'adénocarcinome du poumon (Fowler et al., 2012 ; Stanford et al., 2009). 
Afin de comprendre le mécanisme d'action des effets anticancéreux de cette immunothérapie, une recherche a été réalisée qui a démontré sa capacité à stimuler indirectement une réponse anti-tumorale chez les lymphocytes $\gamma \delta \mathrm{T}$ humains, de telles cellules sont connues par leur rôle dans l'immuno-surveillance protectrice contre le cancer en présentant une cytotoxicité puissante contre les cellules tumorales d'un large éventail de tumeurs malignes épithéliales et hématogènes. Elles produisent également de l'interféron (IFN- $\gamma$ ) et le facteur de nécrose tumorale (TNF $\alpha$ ) en réponse aux mycobactéries et à la tumeur, qui potentialisent les réponses immunitaires protectrices à médiation cellulaire contre le cancer. En effet, la préparation mycobactérienne stimule les cellules dendritiques myéloïdes circulantes de types 1 qui synthétisent par la suite les cytokines IL-12, IL- $1 \beta$ et le TNF $\alpha$. Celles ci induisent l'activation des lymphocytes $\gamma \delta \mathrm{T}$ qui entraînent, par conséquent, la production de $\mathrm{TH} 1$, la régulation à la hausse de la granzyme $\mathrm{B}$ et une cytotoxicité accrue envers les cellules tumorales (Fowler et al., 2012).

\section{3 - Psoriasis}

De nouveaux traitements pour le psoriasis sont en cours de développement, mais beaucoup sont associée à une efficacité limitée, à des effets secondaires ou à une récurrence rapide. Ainsi, le but des nouveaux agents est d'induire des rémissions à plus long terme avec moins d'effets indésirables. Dans ce cadre, il a été démontré qu'une suspension de $M$. vaccae, tuée par la chaleur et administrée par voie intradermique, peut induire des périodes de rémission chez certains patients atteints de psoriasis. Pour évaluer davantage l'efficacité de $M$. vaccae chez les patients atteints de psoriasis modéré à sévère, une autre investigation a été menée dans laquelle 24 patients ont reçu deux inoculations intradermiques de $M$. vaccae dans une peau deltoïde sans lésion, séparées par une période de 3 semaines.

Douze semaines après le début du traitement, 14 patients sur 24 (soit $58 \%$ ) ont présenté une amélioration marquée (réduction du score de l'indice de la sévérité de la maladie de plus de 50\%), deux ont eu une amélioration modérée (réduction de 25-50\%), six ont resté inchangés (réduction de 25\%), et deux ont vu leurs cas empirés (augmentation de> 5\%). À 24 semaines, 11 des 22 patients ont continué à montrer une amélioration de plus de 50\%. Cinq patients ont eu une clairance complète des lésions cutanées qui ont duré au moins 6 mois.

Par conséquent, l'administration intradermique de la suspension de $M$. vaccae tuée par la chaleur a été bien tolérée et a induit une amélioration cliniquement significative chez la majorité des patients atteints de psoriasis dans cette cohorte (Balagon et al., 2000).

Une autre recherche effectuée par Lehrer et ses collaborateurs (1998) a montré que l'immunothérapie avec une injection de $M$. vaccae, tuée à la 
chaleur et suspendue dans une solution saline tamponnée au borate (10 $\mathrm{mg} / \mathrm{ml}$ ) chez 31 patients atteints de psoriasis chronique en plaques, procure un bénéfice clinique durable et elle peut être ainsi un traitement sûr et efficace pour de nombreux malades, avec des effets secondaires minimes.

Selon Dalbeth et al., (2004), il semble que l'immunothérapie à Mycobacterium dans le psoriasis implique une augmentation des taux d'IL-10 après l'injection de PVAC, une préparation à la base de Mycobacterium vaccae tuée par la chaleur délipidée et déglycolipidée. Ce qui pourrait entraîner une amélioration du psoriasis en raison de l'inhibition de la fonction des lymphocytes $\mathrm{T}$ et de la réduction de la production d'IFN-gamma et de TNF-alpha (Dalbeth et al., 2004). Effectivement, cette modification dans la production de cytokines peut être pertinente dans le traitement de la maladie en question pour maintes raisons : il a été démontré que l'IFN-gamma, synthétisé principalement par les cellules $\mathrm{T}$, pourrait jouer un rôle dans la pathogenèse du psoriasis. En fait, lorsqu'il est injecté dans la peau de sujets normaux, l'IFN-gamma peut induire une hyperplasie des kératinocytes et des lésions similaires aux plaques psoriasiques (Dalbeth et al., 2004). Tandis que, le TNF-alpha joue un rôle central dans le développement des plaques de psoriasis via un ensemble des phénomènes, parmi lesquels figurent, l'activation de la voie $\mathrm{NF}-\kappa \mathrm{B}$ étant responsable de la production de nombreuses molécules inflammatoires induisant une cascade d'activation cellulaire, en particulier, la stimulation de la migration des cellules dendritiques vers le derme et les ganglions ainsi que l'induction d'une prolifération des lymphocytes T et des kératinocytes (Rozieres et al., 2006). Par ailleurs, l'IL10, induite par la PVAC, est un inhibiteur connu du TNFalpha, ce qui pourrait, éventuellement, avoir un effet bénéfique sur le psoriasis en réduisant la production de TNF-alpha (Dalbeth et al.2004).

\section{4 - Stress et anxiété}

Précédemment, il a été rapporté que les propriétés immunorégulatrices et anti-inflammatoires de $M$. vaccae confèrent une résistance au stress chez la souris (Fox et al., 2017). Dans le même cadre, une étude a été menée en montrant que l'immunisation avec $M$. vaccae des souris stressées a induit des effets anxiolytiques ou de réduction de la peur tels que mesurés sur le labyrinthe (Reber et al., 2016). Plus pertinent encore, il a été rapporté, récemment, par une étude exécutée par Loupy et al., (2019) que des sources extra-hypothalamiques (noyau du lit de la strie terminale et noyau central de l'amygdale ) de la sécrétion de l'hormone de libération de la corticotropine, un neuropeptide associé à des troubles liés au traumatisme et stress, favorisent les comportements liés au stress, y compris l'anxiété et la peur chez les rats. Du temps que, la pré-immunisation avec $M$. vaccae NCTC 11659 favorise la résistance au stress en atténuant l'expression de l'ARNm de 
neuropeptide dans les régions précitées chez ces animaux exposés à un paradigme de sursaut potentialisé par la peur. Par ailleurs, il a été mis en exergue, antérieurement, qu'une augmentation de la production de cette hormone pourrait être associée à une réponse immunitaire hyperactive ou dysrégulée chez les personnes atteintes de troubles liées au stress. Ces résultats suggèrent ainsi que $M$. vaccae pourrait fonctionner par un mécanisme qui favorise simultanément l'immunorégulation et diminue les comportements coordonnés par l'hormone de libération de la corticotropine (Loupy et al., 2019).

D'un autre coté, dans une autre recherche menée par Matthews et Jenks (2013), il a été prouvé que les souris nourries des cellules vivantes de M. vaccae avant et pendant une tâche d'apprentissage labyrinthe ont révélé une réduction des comportements liés à l'anxiété et du temps de labyrinthe, testées à trois niveaux de difficulté de labyrinthe sur 12 essais pendant quatre semaines. En effet, les souris traitées ont achevé le labyrinthe deux fois plus vite que les témoins, et ont réduit les comportements liés à l'anxiété. Dans une série consécutive de 12 essais de labyrinthe sans exposition à $M$. vaccae, les souris traitées ont continué à courir plus vite dans le labyrinthe pendant les trois premiers d'essais, avec moins d'erreurs dans l'ensemble, suggérant une persistance du traitement d'environ une semaine. Après une interruption de trois semaines, un dernier labyrinthe n'a révélé aucune différence entre les expérimentaux et les contrôles. Ces résultats suggèrent un effet bénéfique de M. vaccae vivante sur les comportements liés à l'anxiété et la performance du labyrinthe (Matthews et Jenks., 2013).

Ensemble ces données fournissent un cadre pour élaborer des stratégies efficaces fondées sur l'immuno-régulation par $M$. vaccae pour prévenir ou traiter les pathologies liées au stress et à l'anxiété.

\section{5 - Tuberculose}

Ces dernières années, $M$. vaccae a été utilisée comme un adjuvant d'immunothérapie pour le traitement de la tuberculose. En outre, elle a moins d'effets secondaires. Des études réalisées chez la souris et chez l'homme ont montré que $M$. vaccae pouvait renforcer l'immunité cellulaire spécifique à l'antigène de $M$. tuberculosis (Xu et al., 2009). Effectivement, M. vaccae a été injecté à des souris infectées par M. tuberculosis. La dose optimale de souris traitées à $M$. vaccae, de l'ordre de 22,5 $\mu \mathrm{g} /$ souris, a montré un indice de changement pathologique, un indice de poids de la rate, un indice de poids pulmonaire et un nombre de $M$. tuberculosis plus faibles que ceux du groupe non traité. Le traitement avec $M$. vaccae a augmenté les pourcentages de lymphocytes $\mathrm{T} \mathrm{CD}^{+}$et $\mathrm{CD}^{+}$, de $1^{\prime}$ interféron- $\gamma$, de cellules immunitaires innées incluant les cellules natural killer (NK). Par conséquent, M. vaccae pourrait protéger les souris contre l'infection par $M$. tuberculosis et améliorer 
l'immunité à médiation cellulaire innée et adaptative de la souris, suggérant que $M$. vaccae est un agent immunothérapeutique potentiel dans la tuberculose pulmonaire (Xu et al., 2009). Un constat similaire a été rapporté par l'étude menée par Zhang et al., 2016 montrant que des injections contenant $M$. vaccae tuée par la chaleur peut provoquer une augmentation des réponses de lymphocytes $\mathrm{T}$ régulateurs et $\mathrm{T}$ auxiliaires de type Th1, ce qui peut protéger l'hôte contre l'infection tuberculeuse (Zhang et al., 2016).

Plus intéressant encore, une autre investigation a mis en évidence qu'une combinaison de chimiothérapie antibactérienne et d'immunothérapie avec $M$. vaccae devrait conduire à un développement moins important de la résistance bacillaire aux antituberculeux et à des schémas de traitement sensiblement plus courts (Stanford et Stanford, 2012).

Afin de déterminer les antigènes de $M$. vaccae impliqués dans l'immunothérapie de la tuberculose, une étude surprenante a révélé que huit protéines immunogènes de $M$. vaccae présentent des signaux réactifs spécifiques forts sur les Western blots de sérum de patients infectés par $M$. tuberculosis. En outre, la réponse immunitaire cellulaire a été examinée mettant en évidence ainsi une protéine qui présente un niveau d'immunité cellulaire plus élevé chez les patients atteints de tuberculose pulmonaire. Cette protéine est nommée, MYVA_1927, une aminoglycoside phosphotransferase. Des études complémentaires sur l'antigénicité de la protéine devraient être poursuivies, ce qui pourrait contribuer à la compréhension du mécanisme de l'immunothérapie à $M$. vaccae (Zheng et al., 2017).

\section{Conclusion}

D'après cette présente étude bibliographique, il convient de constater que $M$. vaccae est un modulateur immunitaire puissant et un activateur utile dans la prévention et le traitement de nombreuses maladies dans lesquelles l'immunité joue un grand rôle. Citant, par exemple, le succès remarquable de la thérapie par $M$. vaccae dans différents types de cancer qui a souligné son potentiel en tant qu'agent immuno-thérapeutique contre le cancer ou en tant qu'adjuvant à des chimiothérapies existantes (Fowler et al., 2012 ; Stanford et Stanford, 2012). Plus intéressant encore, des études ont prouvé que l'administration de $M$. vaccae a eu un effet d'amélioration sur les caractéristiques histopathologiques des voies respiratoires dans un modèle d'asthme murin, du temps que, les corticoïdes sont inefficaces pour inverser ce remodelage structurel (Yazi et al., 2007, 2008 ; Akkoc et al., 2015). Par ailleurs, la plupart des investigateurs ont évalué les effets bénéfiques de $M$. vavace sur des modèles animaux, et sur la base de leurs résultats pertinents, ces observations pourraient d'être traduites en études humaines par des essaies cliniques afin mettre en place des stratégies efficaces fondées sur 
l'immunorégulation par $M$. vaccae pour prévenir ou traiter les pathologies liées au dysfonctionnement de système immunitaire avec moins d'effets secondaires sur la santé et plus d'efficacité. Ainsi, $M$. vaccae avec ses composants pourraient transformer le visage de la médecine moderne. Pour cette fin, ce microorganisme mérite d'être exploité amplement par la réalisation d'autres travaux qui s'intéressent à la compréhension approfondie des mécanismes d'action impliquées dans la relation $M$. vaccae et hôte à l'état normal et à l'état pathologique jumelés avec des études visant le criblage des meilleurs modes d'administration qui donnent de bons résultats selon la maladie ciblée sur des modèles animaux et des essaies cliniques ainsi que l'évaluation de nombre des essaies de vaccins à employer à base de M. vaccae à court et à long terme sur l'évolution des pathologies. D'autres investigations de toxicité notamment chez des immunodéprimés peuvent être additionnées avant d'avoir l'autorisation de mise sur le marché de $M$. vaccae comme un agent bio-thérapeutique.

En définitive, le message à transmettre à travers ce travail, c'est que le contact avec la nature, en particulier le sol, présente de nombreux avantages pour notre santé, notamment le renforcement du système immunitaire.

\section{References:}

1. Al-Alawi M., Hassan T., Chotirmall S H., (2014). Transforming growth factor $\beta$ and severe asthma: A perfect storm. Respir Med., 108: $1409-1423$

2. Akkoc T., Ozdemir C., Yazi D., Yesil O., Bahceciler N N., Barlan I., (2015). Mycobacterium vaccae Immunization to Pregnant BALB/c Mice Ameliorated Lung Histopathology and Bone Marrow Eosinophila in Ovalbumin Sensitized Offsprings. Open Journal of Immunology, 4: 31-41.

3. Balagon MV, Walsh DS, Tan PL, Cellona RV, Abalos RM, Tan EV, Fajardo TT, Watson JD, Walsh GP., (2000). Improvement in psoriasis after intradermal administration of heat-killed Mycobacterium vaccae. Int J Dermatol. 39(1):51-8

4. Bjermer L., Diamant Z., (2004). Current and emerging nonsteroidal anti-inflammatory therapies targeting specific mechanisms in asthma and allergy. Treat Respir Med, 3(4):235-246

5. Boenickse R, Juhasz SE. (1964). Beschreibung der neuen Species Mycobacterium vaccae no. sp. Zentralbl. Bakteriol. Orig. 192:133135.

6. Camporota L., Corkhill A., Long H., Lordan J., Stanciu L., Tuckwell N., Cross A., Stanford J.L., Rook G.A., Holgate S.T., Djukanovic R., (2003). The effects of Mycobacterium vaccae on allergen-induced airway responses in atopic asthma. Eur. Respir. J. 21: 287-293. 
7. Cananzi Ferdinando CM, Mudan S., Dunne M., Belonwu N., G Dalgleish AG., (2013). Long-term survival and outcome of patients originally given Mycobacterium vaccae for metastatic malignant melanoma. Hum Vaccin Immunother. 9(11): 2427-2433.

8. Dalbeth N., Yeoman S., Dockerty J.L., Highton J., Robinson E., Tan P.L., Herman D., McQueen F., (2004). A randomized placebo controlled trial of delipidated, deglycolipidated Mycobacterium vaccae as immunotherapy for psoriatic arthritis. Ann Rheum Dis., 63(6):718-722.

9. Fowler D W., Copier J., Wilson N., Dalgleish A G., Bodman-Smith M D., (2012). Mycobacteria activate $\delta \gamma$ T-cell anti-tumour responses via cytokines from type 1 myeloid dendritic cells: a mechanism of action for cancer immunotherapy, Cancer Immunol Immunother, 61:535-547

10. Fox JH., Hassell JE Jr., Siebler PH., Arnold MR., Lamb AK., Smith DG., Day HEW., Smith TM., Simmerman EM., Outzen AA., Holmes KS., Brazell CJ., Lowry CA., (2017). Preimmunization with a heatkilled preparation of Mycobacterium vaccae enhances fear extinction in the fear-potentiated startle paradigm. Brain Behav Immun, 66:7084.

11. Halwani R., Al-Muhsen S., Al-Jahdali H., Hamid Q., (2011). Role of Transforming Growth Factor- $\beta$ in Airway Remodeling in Asthma. Am J Respir Cell Mol Biol, 44: 127-133.

12. Ho Y.S., Adroub S.A., Abadi M., Al Alwan B., Alkhateeb R., Gao G., Ragab A., Shahjahan A., van Soolingen, D., Bitter W., Pain A., Abdallah A.M., 2012, Complete Genome Sequence of Mycobacterium vaccae Type Strain ATCC 25954, J. Bacteriol., 194 (22): 6339-6340.

13. Kikkawa Y., Sugiyama K., Obara K., Hirata H., Fukushima Y., Toda M., Fukuda T., (2012). Interferon-alpha inhibits airway eosinophila and hyperresponsiveness in an animal asthma model. Asia Pac Allergy, 2:256-263

14. Le Bert N., Chain BM., Rook G., Noursadeghi M., (2011). DC Priming by $M$. vaccae Inhibits Th2 Responses in Contrast to Specific TLR2 Priming and Is Associated with Selective Activation of the CREB Pathway. PLoS ONE 6(4): e18346.

15. Lehrer A., Bressanelli A., Wachsmann V., Bottasso O., Bay ML., Singh M., Stanford C., Stanford J., (1998). Immunotherapy with Mycobacterium vaccae in the treatment of psoriasis, FEMS Immunol Med Microbiol., 21: 71-77

16. Li C., Jiang X., Luo M., Feng G., Sun Q., Chen Y., 2016: Mycobacterium vaccae Nebulization Can Protect against Asthma in 
Balb/c Mice by Regulating Th9 Expression. PLoS ONE, 11 (8): e0161164.

17. Loupy, K.M., Arnold, M.R., Hassell, J.E. Jr., Lieb, M.W., Milton, L.N., Cler, K.E., Fox, J.H., Siebler, P.H., Schmidt, D., Noronha, S.I.S., Day, H.E.W., Lowry, C.A., (2019). Evidence that preimmunization with a heat-killed preparation of Mycobacterium vaccae reduces corticotropin-releasing hormone mRNA expression in the extended amygdala in a fear-potentiated startle paradigm, Brain Behav. Immun., 77:127-140

18. Martinelli R., Adams V. C., Rook G. A. W., Brunet L. R., (2004). Effects of Mycobacterium vaccae on toll-like receptor expression. J Allergy Clin Immunol Abstracts, 113 (2): S187

19. Matthews D.M., Jenks S.M., (2013). Ingestion of Mycobacterium vaccae decreases anxiety-related behavior and improves learning in mice, Behav Processes. 96: 27-35.

20. O’Brien M.E., Anderson H., Kaukel E., O’Byrne K., Pawlicki M., von Pawel J., Reck M., (2004). SRL172 (killed Mycobacterium vaccae) in addition to standard chemotherapy improves quality of life, without affecting survival in patients with advanced, non-small-cell lung cancer: phase III results, Ann. Oncol., 15: 906-914.

21. Patel P.M., Sim S., O’Donnell D.O., Protheroe A., Beirne D., Stanley A., et al., (2008). An evaluation of a preparation of Mycobacterium vaccae (SRL172) as an immunotherapeutic agent in renal cancer, Eur. J. Cancer, 44 (2008) 216-223.

22. Racila DM., Kline JN., (2005). Perspectives in asthma: molecular use of microbial products in asthma prevention and treatment. . J Allergy Clin Immunol. 116(6):1202-5.

23. Reber SO, Siebler PH, Donner NC, Morton JT, Smith DG, Kopelman JM, Lowe KR, Wheeler KJ, Fox JH, Hassell JE Jr, Greenwood BN, Jansch C, Lechner A, Schmidt D, Uschold-Schmidt N, Füchsl AM,Langgartner D, Walker FR, Hale MW, Lopez Perez G, Van Treuren W, González A, Halweg-Edwards AL, Fleshner M, Raison CL, Rook GA, Peddada SD, Knight R, Lowry CA, (2016). Immunization with a heat-killed preparation of the environmental bacterium Mycobacterium vaccae promotes stress resilience in mice, Proc Natl Acad Sci U S A. 113(22): 1-10

24. Rook G.A.W., Hamelmannb E., Brunet L. R., (2007). Mycobacteria and allergies, Immunobiology, 212: 461-473.

25. Rozieres A., Hennino A., Nicolas J.-F., (2006). Le TNF-alpha dans la physiopathologie du psoriasis. Ann Dermatol Venereol, 133 : 173-180.

26. Shirtcliffe P.M., Goldkorn A., Weatherall M., Tan P.L., Beasley R., (2003). Pilot study of the safety and effect of intranasal delipidated 
acid-treated Mycobacterium vaccae in adult asthma. Respirology, 8: 497-503.

27. Stanford J., Stanford C., (2012): Mycobacteria and their world, Int. J. Mycobacteriol I: 3-12.

28. Stanford J.L., Stanford C.A., O’Brien M.E.R, Grange J.M., (2008). Successful immunotherapy with Mycobacterium vaccae in the treatment of adenocarcinoma of the lung, Eur. J. Cancer, 44 : 224-227.

29. Stanford J., Stanford C., Dlugovitzky D., Fiorenza G., Martinel-Lamas D., Selenscig D., Bogue C., (2009). Potential for immunotherapy with heat-killed Mycobacterium vaccae in respiratory medicine. Immunotherapy, 1(6):933-947.

30. Xu L., Wang Y., Zheng X., Gui X., Tao L., Wei H., 2009, Immunotherapeutical Potential of Mycobacterium vaccae on $M$. tuberculosis Infection in Mice, Cellular \& Molecular Immunology. 6(1): 67-72.

31. Yazi D., Akkoc T., Ozdemir C., Yesil O., Aydogan M., Sancak R., Bahceciler NN., Barlan IB., (2007). Long-term modulatory effect of Mycobacterium vaccae treatment on histopathologic changes in a murine model of asthma. Ann Allergy Asthma Immunol. 98(6):573589

32. Yazi D., Akkoc T., Yesil O., Ozdemir C., Aydoğan M., Koksalan K., Bahceciler NN., Barlan IB., (2008). Treatment with Mycobacterium vaccae ameliorates airway histopathology in a murine model of asthma. Allergy Asthma Proc. 29(1): 67-73.

33. Zahir I., (2018). Microorganisms producing antimycobacterial substances. First edition, Lambert Academic Publishing. 204 pp. ISBN-13: 978-613-7-37914-1.

34. Zhang L., Jiang Y., Cui Z., Yang W., Yue L., Ma Y., Shi S., Chunfang Wang C., Wang C., Qian A., (2016). Mycobacterium vaccae induces a strong Th1 response that subsequently declines in C57BL/6 mice. J Vet Sci, 17(4): 505-513

35. Zheng J., Chen L., Liu L., Li H., Liu B., Zheng D., Liu T., Dong J., Sun L., Zhu Y., Yang J., Zhang X., Jin Q., (2017). Proteogenomic Analysis and Discovery of Immune Antigens in Mycobacterium vaccae. Mol. Cell. Proteomics. 16.9: 1578 - 1590

36. Zuany-Amorim C., Sawicka E., Manlius C., Le Moine A., Brunet L.R., Kemeny D.M., Bowen G., Rook G., Walker C., (2002). Suppression of Airway Eosinophilia by Killed Mycobacterium vaccae-Induced Allergen-Specific Regulatory T-Cells. Nat. Med., 8: 625-629. 